\title{
Separable states can be used to distribute entanglement
}

\author{
T. S. Cubitt, ${ }^{1}$ F. Verstraete, ${ }^{1}$ W. Dür, ${ }^{2}$ and J.I. Cirac ${ }^{1}$ \\ ${ }^{1}$ Max Plank Institut für Quantenoptik, Hans-Kopfermann Str. 1, D-85748 Garching, Germany \\ ${ }^{2}$ Sektion Physik, Ludwig-Maximilians-Universität München, Theresienstr. 37, D-80333 München, Germany
}

(Dated: February 21, 2003)

\begin{abstract}
We show that no entanglement is necessary to distribute entanglement; that is, two distant particles can be entangled by sending a third particle that is never entangled with the other two. Similarly, two particles can become entangled by continuous interaction with a highly mixed mediating particle that never itself becomes entangled. We also consider analogous properties of completely positive maps, in which the composition of two separable maps can create entanglement.
\end{abstract}

PACS numbers: 03.67.Mn, 03.67.-a

Einstein associated entanglement with "spooky actionat-a-distance", a strange quantum effect that did not tally with his ideas of how the universe ought to work [1]. Modern quantum information theory, however, takes a different view: entanglement is a physical quantity. And like other physical quantities, it can be used as a resource. The major successes in the field have come from asking: "what new possibilities arise when entanglement is available?". The power of this quantum resource became especially apparent after the discovery of quantum teleportation 2], which showed that, if entanglement and classical communication are available, global quantum operations can be implemented locally. Since then, huge progress has been made in describing the way entanglement can be distributed and manipulated among separated parties. As for other physical quantities, conservation laws have been formulated dictating e.g. that the amount of entanglement can not be increased by local operations and classical communication (LOCC) 3]. Specifically, this means entanglement can only be created by an interaction between particles.

In this Letter, we investigate more closely the conditions required to entangle two distant particles. Though there is no unique way to quantify entanglement for mixed states, the usual definition of an entangled state - as one that can not be created by LOCC - is unambiguous. To create entanglement, then, a global quantum operation is necessary. For separated particles, this must be carried out by sending a mediating particle between them (see Fig. 1), i.e. by communication via a quantum channel. Note that fundamentally this is the only way entanglement is created 19], as all interactions in nature occur via mediating gauge bosons. It is clear that the particles can be entangled if the mediating (or 'ancilla') particle becomes entangled: entangle the first particle with the ancilla, send the latter through the channel, and swap it with the second particle. We can picture the entanglement being sent through the channel. One would expect that the ancilla necessarily becomes entangled, in any scheme. Surprisingly, we will prove that this intuitive picture is false, and that two particles can become entangled without the ancilla ever becoming entangled. (Note that this does not imply entanglement can be created by LOCC since a quantum channel was used.)

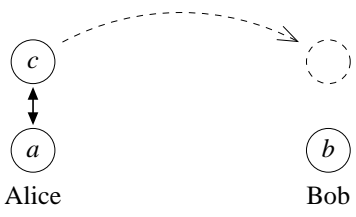

(a)

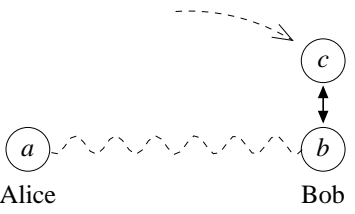

(b)
FIG. 1: Alice and Bob each have a particle they wish to entangle with the other. (a) Alice interacts an ancilla $c$ with her particle $a$, sends $c$ to Bob, (b) who interacts $c$ with his particle $b$. At the end they share some entanglement. Surprisingly, $c$ does not have to become entangled with $a$ and $b$.

We demonstrate this fact in two different ways. First we consider a process in which two particles interact continuously with an ancilla (Fig. 21). We prove that if it is possible to entangle the particles while leaving the ancilla separable at all times, it is possible to turn this into a discretized scheme in which an ancilla is sent between Alice and Bob a number of times. Inspired by quantum optical systems, we give an example of such a continuous process in which the ancilla remains separable.

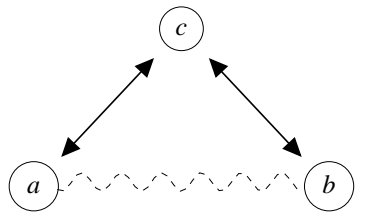

FIG. 2: Particles $a$ and $b$ interact continuously via a mediating particle $c$. For certain initial states, $c$ remains separable from $a$ and $b$ at all times during the evolution of the system, yet at the end $a$ and $b$ are entangled.

As a second demonstration, we give an explicit discrete procedure in which an ancilla is sent from Alice to Bob, and the interactions are described by local unitary operations: a single use of a quantum channel is already sufficient to distribute entanglement without sending entan- 
glement. By analyzing bipartite partitions of the tripartite system, we explain why the process works. Finally, we translate this result describing properties of states into a result describing properties of completely positive maps (CPM): we give an example of two maps that individually cannot create entanglement from initially separable states, nevertheless their composition can.

First consider the continuous case. As a model of an interaction mediated by another particle, consider a system of two particles ' $a$ ' and ' $b$ ' both interacting with the same ancilla ' $c$ ' (Fig. (2) but not directly with each other. If we restrict ourselves to pure states, the effect presented above is impossible: to entangle $a$ and $b, c$ must become entangled with respect to the partition $(a b)-c$ [20]. This is easily proven by looking at the infinitesimal change of a pure separable state $|a\rangle|b\rangle|c\rangle$ under the action of a Hamiltonian of the form $H_{A C} \otimes \mathbb{1}_{B}+\mathbb{1}_{A} \otimes H_{B C}$. The condition that the ancilla remains separable is given by

$$
\begin{aligned}
\left(\mathbb{1}+\delta t\left(H_{A C}+\right.\right. & \left.\left.H_{B C}\right)\right)|a\rangle|b\rangle|c\rangle \\
& =\left(|a\rangle|b\rangle+\delta t\left|\psi_{a b}\right\rangle\right)\left(|c\rangle+\delta t\left|\psi_{c}\right\rangle\right)
\end{aligned}
$$

where $\left|\psi_{a b}\right\rangle$ and $\left|\psi_{c}\right\rangle$ are unnormalized states. It is readily seen that the state $|a\rangle|b\rangle+\delta t\left|\psi_{a b}\right\rangle$ is entangled to first order in $\delta t$ if and only if $\left|\psi_{a b}\right\rangle$ has a non-vanishing component $\left\langle a^{\perp}\right|\left\langle b^{\perp} \mid \psi_{a b}\right\rangle$ for some $\left|a^{\perp}\right\rangle,\left|b^{\perp}\right\rangle$ orthogonal to $|a\rangle,|b\rangle$. However, multiplying equation (1) by $\left\langle a^{\perp}\right|\left\langle b^{\perp}\right|$ shows this is not the case. This is in complete accordance with the intuition that entanglement can only be transmitted if the mediating particle itself becomes entangled.

Let us however investigate how the amount of entanglement of the ancilla is related to the amount of entanglement that can be transmitted. Physically, the ancilla has a spectrum of energy levels corresponding to eigenstates of its Hamiltonian. Imagine that interactions with particles $a$ and $b$ couple eigenstates of the ancilla, and the interactions are weak so that transitions between energy levels are virtual. The most significant processes in the evolution of the system are then second order virtual transitions of $c$ from an energy level back to itself, accompanied by an interaction between particles $a$ and $b$. If the ancilla starts in an energy eigenstate, the virtual transition will leave it in the same eigenstate, not entangled with $a$ or $b$. It will become entangled with $a$ or $b$ due to less significant higher order processes, but this entanglement will be weak. Particles $a$ and $b$, however, can become strongly entangled if the interaction lasts a long time, and we have a situation close to the advertised effect: $a$ and $b$ can become entangled while the ancilla remains almost separable. But assume now that there is noise in the system, which can be modeled by mixing the quantum states with the maximally mixed state. Mixing a barely entangled state with an amount of noise proportional to the entanglement destroys the entanglement [4, [5]. We can therefore add a sufficiently large weight of the maximally mixed state to remove the weak entanglement with the ancilla, but not so large that it destroys the strong entanglement between $a$ and $b$. (This is related to the results that entanglement can be created by interaction with a common heat bath $\underline{6}$ ]). If mixed states are considered, it therefore seems to be possible to obtain the desired effect in the continuous regime.

But this implies the seemingly stronger result that a separable ancilla bouncing back and forth between two distant particles (see Fig. 1) can distribute (distillable) entanglement. A continuous interaction whose evolution operator is of the form $e^{-i\left(H_{A}+H_{B}\right) t}$ can be discretized, e.g. using the Trotter expansion: $e^{A+B}=$ $\lim _{n \rightarrow \infty}\left(e^{A / n} e^{B / n}\right)^{n}$. Note that the discretized evolution can be made arbitrary close to the original one by choosing a large (but finite) $n$. This strange effect forces us to completely abandon the physical picture of sending entanglement through a quantum channel. E.g. this implies that the Ekert protocol in quantum cryptography [7] could be implemented by sending separable states.

To demonstrate these ideas, let us consider a specific example of the continuous case, in which $a$ and $b$ are qubits and the ancilla $c$ is a qutrit with eigenstates $|0\rangle$, $|1\rangle$ and $|2\rangle$. Motivated by ion-trap quantum computation experiments (see e.g. Ref. [8]), in which vibrational modes of the trapped ions play the role of the ancilla 9,10 , or by cavity-QED experiments [11] in which the ancilla is the cavity mode, we consider the Hamiltonian

$$
H=\hat{c}^{\dagger} \hat{c} \otimes \mathbb{1}+\frac{\epsilon}{2}\left(\hat{c}+\hat{c}^{\dagger}\right) \otimes\left(\sigma_{x}^{a}+\sigma_{x}^{b}\right),
$$

where $\epsilon$ is small and characterizes the strength of the interaction, and $\hat{c}^{\dagger}$ is the "creation" operator in the truncated Hilbert space of the ancilla: $\hat{c}=|0\rangle_{c}\langle 1|+\sqrt{2}| 1\rangle_{c}\langle 2|$, so $\hat{c}^{\dagger} \hat{c}=|1\rangle_{c}\langle 1|+2| 2\rangle_{c}\langle 2|=H_{0}$. The interactions couple eigenstates of the ancilla, as described above. As we are interested in the leading order evolution (and how much the true evolution deviates from this), we turn to perturbation theory. The Hamiltonian operates in four invariant subspaces: $H=H_{+}^{c} \otimes \Pi_{++}^{a b}+H_{-}^{c} \otimes \Pi_{--}^{a b}+H_{0}^{c} \otimes$ $\left(\Pi_{+-}^{a b}+\Pi_{-+}^{a b}\right)$, where $H_{ \pm}=\hat{c}^{\dagger} \hat{c} \pm \epsilon\left(\hat{c}+\hat{c}^{\dagger}\right)$, and the $\Pi^{\prime} \mathrm{s}$ denote projectors whose subscripts refer to the $|+\rangle,|-\rangle$ qubit states with $| \pm\rangle=1 / \sqrt{2}(|0\rangle \pm|1\rangle)$. The evolution operator can then be written as a direct sum of operators on these spaces: $U=e^{-i H_{+} t} \oplus e^{-i H_{0} t} \oplus e^{-i H_{0} t} \oplus$ $e^{-i H_{-} t}$. Expanding $H_{ \pm}$using standard perturbation theory, we have $e^{-i H_{ \pm} t}=e^{-i D t}+O\left(\epsilon^{4} t\right)+O(\epsilon)$, where $D=\operatorname{diag}\left(-\epsilon^{2}, 1-\epsilon^{2}, 2+2 \epsilon^{2}\right)$ is the matrix of eigenvalues of $H_{ \pm}$approximated to leading order (accurate to $3^{\text {rd }}$ order since all odd order terms are zero). The $O\left(\epsilon^{4} t\right)$ term arises from higher order perturbations of the eigenvalues, the $O(\epsilon)$ term from perturbation of the eigenstates. We can then approximate the evolution operator:

$$
U=U_{\mathrm{eff}}+O\left(\epsilon^{4} t\right)+O(\epsilon)
$$

where $U_{\text {eff }}=e^{-i D t} \oplus e^{-i H_{0} t} \oplus e^{-i H_{0} t} \oplus e^{-i D t}$. In the limit of small $\epsilon$, the evolution of the system is "close" to 
evolution under $U_{\text {eff }}$. How do states evolve under $U_{\text {eff }}$ ? If we start $c$ in an eigenstate of $U_{\text {eff }}(|0\rangle,|1\rangle$ or $|2\rangle)$, its state remains unchanged up to a global phase, and can not become entangled with $a$ and $b$. If $a$ and $b$ start in a superposition of eigenstates of $U_{\text {eff }}$, the $|++\rangle$ and $|--\rangle$ portions acquire a phase difference relative to the $|+-\rangle$ and $|-+\rangle$ portions $\left(e^{-2 i \epsilon^{2} t}\right.$ if $c$ is in the $|2\rangle$ state, $e^{i \epsilon^{2} t}$ otherwise, recalling the expressions for $D$ and $H_{0}$ ). Particles $a$ and $b$ can therefore become highly entangled for times of order $1 / \epsilon^{2}$, even though the ancilla remains separable at all times. The true evolution under $U$ deviates from that under $U_{\text {eff }}$ due to the higher order terms in Eq. (2), and will entangle the ancilla if the system starts in a pure state. To show that a small amount of mixing is sufficient to remove this entanglement while leaving $a$ and $b$ highly entangled, we must bound this deviation at times $t \sim 1 / \epsilon^{2}$ to $O(\epsilon)$ (that is, the deviation between what we would get if the system evolved under $U_{\text {eff }}$, and what we $d o$ get when the system evolves under $U$ ). This means bounding the matrix norm $\left\|e^{-i H_{ \pm} t}-e^{-i D t}\right\|$. To achieve this, we make use of the matrix of eigenvectors of $H_{ \pm}$approximated to third order in $\epsilon$, denoted $X$. Then

$$
\begin{aligned}
\left\|e^{-i H_{ \pm} t}-e^{-i D t}\right\| \leq\left\|e^{-i H_{ \pm} t}-X e^{-i D t} X^{-1}\right\| \\
+\left\|X e^{-i D t} X^{-1}-e^{-i D t}\right\| .
\end{aligned}
$$

The first norm on the right hand side $\left(\mathcal{N}_{1}\right)$ is governed by higher order perturbations of the eigenvalues, and can be bounded to $O\left(\epsilon^{4} t\right)$ by standard results in linear algebra [12, $§ 2.3 .4 \& \S 11.3 .2]$, giving

$$
\mathcal{N}_{1} \leq\left\|X^{-1}\right\|^{2}\|X\|\left\|H_{ \pm} X-X D\right\| t .
$$

The second norm $\left(\mathcal{N}_{2}\right)$ is governed by perturbation of the eigenvectors. It can be bounded to $O(\epsilon)$ by expanding the exponentials as power series, and making use of the fact that a matrix satisfies its own characteristic equation to reduce the expansions to finite order polynomials, whose coefficients are functions of the eigenvalues $d_{1, \ldots, 3}$ of $D$ and time $t$. The time dependence is removed by taking maxima of these coefficients over all times, giving

$$
\begin{aligned}
\mathcal{N}_{2} \leq\left\|X^{-1}\right\| \cdot \frac{2}{|\Delta|}( & \left(d_{3}^{2}-d_{1}^{2}\right)\|X D-D X\| \\
& \left.+\left(d_{3}-d_{1}\right)\left\|X D^{2}-D^{2} X\right\|\right),
\end{aligned}
$$

where $\Delta$ is the determinant of the Vandermonde matrix formed by $d_{1, \ldots, 3}$.

We can now investigate the evolution of the initial state

$$
\rho=\frac{1}{(1+\alpha)^{2}}\left(|0\rangle_{a}\langle 0|+\alpha \frac{1}{2} \mathbb{1}_{a}\right) \otimes\left(|0\rangle_{b}\langle 0|+\alpha \frac{1}{2} \mathbb{1}_{b}\right) \otimes \frac{1}{3} \mathbb{1}_{c}
$$

which is not only separable, but also classically uncorrelated. The ancilla is in a mixture of eigenstates of $U_{\text {eff }}$, so will not become entangled to leading order (i.e. by $U_{\text {eff }}$ ), whereas $a$ and $b$ are in a superposition of the eigenstates of $U_{\text {eff }}(|+\rangle,|-\rangle)$ and do become entangled, as discussed previously. Using the bounds $\mathcal{N}_{1,2}$, we can bound the contribution of the higher order terms to the the evolved density matrix. This allows us to derive an expression for the minimum amount of mixing $\alpha$ that is required, for a given $\epsilon$, to ensure any entanglement with the ancilla is removed. We derive a second inequality involving $\alpha$ and $\epsilon$ by considering the maximum amount of mixing that does not destroy the entanglement between $a$ and $b$ [13]. We can then calculate a bound on the $\epsilon$ that allow the two expressions to be satisfied simultaneously, i.e. the interaction strengths for which there is some $\alpha$ that removes all entanglement with the ancilla but leaves entanglement between $a$ and $b$. We obtain the condition $\epsilon<1 / 7000$. We have therefore proven that the desired effect is physical. Numerically we find that this bound is much too strict, and $\epsilon \lesssim 1 / 10$ is already sufficient.

Let us now move to the discrete case (see Fig. (1). Alice and Bob start by preparing a (classically correlated) separable state in step 1, where Alice has particles $a$ and $c$, Bob has particle $b$. In step 2, Alice applies an operation on her two particles $a$ and $c$, then sends particle $c$ to Bob. Finally, in step 3 Bob applies an operation on $b$ and $c$, resulting in a state that contains (distillable) entanglement between $a$ and $b$ (tracing out $c$ ), even though $c$ has remained separable from $(a b)$ throughout.

This quantum information picture gives us more insight into the basic principles underlying this (bipartite) effect, in which entanglement properties of tripartite systems turn out to play a key role. In tripartite systems, the entanglement properties of all bipartite partitions are independent [14]. For example, there exist mixed states in which $c$ is separable from $(a b), b$ is separable from $(a c)$, yet $a$ is entangled with $(b c)$. Indeed, step 2 creates a state with precisely these properties. Step 3 then entangles $b$ with $(a c)$ without changing the entanglement properties of the other bipartite partitions. At each step, $c$ remains separable from the rest of the system.

We now give an explicit example in which all particles are qubits, demonstrating the above process. Step 1: Alice and Bob prepare the (manifestly separable) classically correlated state

$\rho_{a b c}=\frac{1}{6} \sum_{k=0}^{3}\left|\Psi_{k}, \Psi_{-k}, 0\right\rangle\left\langle\Psi_{k}, \Psi_{-k}, 0\left|+\sum_{i=0}^{1} \frac{1}{6}\right| i, i, 1\right\rangle\langle i, i, 1|$

where $\left|\Psi_{k}\right\rangle=1 / \sqrt{2}\left(|0\rangle+e^{i k \pi / 2}|1\rangle\right)$. Step 2: Alice applies a CNOT operation on particles $a$ and $c$ (where $a$ is the control qubit), producing the state

$$
\sigma_{a b c}=\frac{1}{3}\left|\Psi_{G H Z}\right\rangle\left\langle\Psi_{G H Z}\right|+\sum_{i, j, k=0}^{1} \beta_{i j k} \Pi_{i j k}
$$

where $\left|\Psi_{G H Z}\right\rangle_{a b c}=1 / \sqrt{2}(|000\rangle+|111\rangle), \quad \Pi_{i j k}=$ $|i j k\rangle\langle i j k|$, and all $\beta$ 's are 0 apart from $\beta_{001}=\beta_{010}=$ 
$\beta_{101}=\beta_{110}=1 / 6$. This state is invariant under a permutation of the qubits $b$ and $c$, and must therefore be separable with respect to the $b-(a c)$ and $c-(a b)$ partitions. Step 3: Bob applies a CNOT on $b$ and $c$ (with $b$ as the control qubit), resulting in the state

$$
\tau_{a b c}=\frac{1}{3}\left|\phi^{+}\right\rangle_{a b}\left\langle\phi^{+}|\otimes| 0\right\rangle_{c}\left\langle 0\left|+\frac{2}{3} \mathbb{1}_{a b} \otimes\right| 1\right\rangle_{c}\langle 1|
$$

where $\left|\phi^{+}\right\rangle=1 / \sqrt{2}(|00\rangle+|11\rangle)$ is maximally entangled. The ancilla $c$ clearly remains separable with respect the rest of the system, but the state now contains entanglement between $a$ and $b$.

Bob can extract this entanglement in a number of ways. Measuring $c$ in the computational basis, he can extract a maximally entangled state of $a b$ with probability $1 / 3$. Alternatively, if a deterministic effect is required, he can apply a local completely positive map (CPM) to particles $b$ and $c$, defined by $\mathcal{E}_{b c}(\rho)=\sum_{j} O_{b c}^{(j)} \rho O_{b c}^{(j) \dagger}$ with Kraus operators $O_{b c}^{(1)}=\mathbb{1}_{b} \otimes|0\rangle_{c}\langle 0|, O_{b c}^{(2)}=$ $|0\rangle_{b}\langle 0|\otimes| 1\rangle_{c}\langle 1|$, and $O_{b c}^{(3)}=|0\rangle_{b}\langle 1|\otimes| 1\rangle_{c}\langle 1|$, satisfying $\sum_{j} O^{(j) \dagger} O^{(j)}=\mathbb{1}$. Throwing away the ancilla leaves the state $\rho_{a b}=\operatorname{tr}_{c}\left(\mathcal{E}_{b c}\left(\tau_{a b c}\right)\right)=1 / 3\left|\phi^{+}\right\rangle\left\langle\phi^{+}\right|+1 / 3 \Pi_{00}+$ $1 / 3 \Pi_{10}$, where $\Pi_{i j}=|i j\rangle\langle i j|$. This has non-positive partial transpose, so must be (distillable) entangled [15, 16]. Thus Alice and Bob achieve the announced effect.

An effect in the same spirit as the examples presented for (mixed) states can also be found for quantum operations, i.e. for trace-preserving completely positive maps (TPCPM). The result is a direct application of the duality between non-local maps and states [17] to the present puzzle. We will prove that a composition of two separable maps can be entangling. More precisely, we consider a first (nonlocal) map acting on three qubits $a b c$ that cannot create entanglement with respect to the bipartite partitions $b-(a c)$ and $c-(a b)$, and a second map that cannot create entanglement with respect to $a-(b c)$ and $c-(a b)$. Obviously, neither of the maps can create entanglement between $a$ and $b$. Interestingly, however, it turns out that the sequential application of these TPCPM's can create (distillable) entanglement between $a$ and $b$.

Let us consider the TPCPM $\mathcal{E}_{1}$ with Kraus operators

$$
\begin{array}{cc}
A_{1}=|000\rangle\langle 000|+| 111\rangle\langle 111|, \\
A_{2}=|001\rangle\left\langle 001\left|, \quad A_{3}=\right| 010\right\rangle\left\langle 010\left|, \quad A_{4}=\right| 101\right\rangle\langle 101|, \\
A_{5}=|110\rangle\left\langle 110\left|, \quad A_{6}=\right| 000\right\rangle\left\langle 011\left|, \quad A_{7}=\right| 111\right\rangle\langle 100| .
\end{array}
$$

Using the duality between maps and states developed in [17], one can easily show that this map cannot create entanglement in the partitions $b-(a c)$ and $c-(a b)$ : the CPM corresponding to Kraus operators $\left\{A_{1} \ldots A_{5}\right\}$ of Eq. (4) gives rise to a dual state that is exactly of the form of Eq. (3), which is separable in the stated partitions. As this state can be used to implement the map probabilistically, the map itself cannot be entangling in $b-(a c)$ or in $c-(a b)$. For the second map, we define the
TPCPM $\mathcal{E}_{2}$ with Kraus operators as for $\mathcal{E}_{1}$, but with the roles of $a$ and $b$ interchanged. This cannot create entanglement in the $a^{-}(b c)$ and $c-(a b)$ partitions.

The map $\mathcal{E}$ obtained by composing both maps $\mathcal{E}=$ $\mathcal{E}_{2} \circ \mathcal{E}_{1}$ has Kraus operators $C_{1}=A_{1}, C_{2}=A_{2}, C_{3}=$ $|000\rangle\left\langle 010\left|, C_{4}=A_{6}, C_{5}=A_{7}, C_{6}=\right| 111\right\rangle\langle 101|, C_{7}=A_{5}$. One can readily prove that this map cannot entangle states in the partition $c-(a b)$, but is entangling in the partitions $a-(b c)$ and $b-(a c)$. It immediately follows that the composition map can create (distillable) entanglement between $a$ and $b$ out of a separable state. For example, an entangled state $\rho_{A B}$ is obtained by acting with $\mathcal{E}$ on the separable state $|+++\rangle$, when the map is followed by a local measurement of $c$ in the $|+\rangle,|-\rangle$ basis.

In this letter we have investigated the conditions required to entangle two distant particles. We have shown that continuous interaction with a highly mixed ancilla can create entanglement without entangling the ancilla. We have also shown that entanglement can be created by sending a separable ancilla, and that an analogous effect exists for quantum operations. These results easily generalize to multi-party systems: many particles interacting with a common ancilla, or an ancilla sent between each party in turn. Thus no entanglement is required to create entanglement, forcing us to abandon the picture of entanglement being sent through a quantum channel.

We thank M. Lewenstein, J. M. Raimond, W. Zurek, and S. Popescu for valuable discussions. This work was supported in part by the E.C. (RESQ IST-200137559, HPMF-CT-2001-01209 (W.D.)) and the Kompetenznetzwerk "Quanteninformationsverarbeitung" der Bayerischen Staatsregierung.

[1] A. Einstein, B. Podolsky, and N. Rosen, Physical Review 47, 777 (1935).

[2] C. H. Bennett et al., Phys. Rev. Lett. 70, 1895 (1993).

[3] C. H. Bennet et al., Phys. Rev. A. 54, 3824 (1996).

[4] G. Vidal and R. Tarrach, Phys. Rev. A. 59, 141 (1999).

[5] S. L. Braunstein et al., Phys. Rev. Lett. 83, 1054 (1999).

[6] D. Braun, Phys. Rev. Lett. 89, 277901 (2002).

[7] A. Ekert, Phys. Rev. Lett. 67, 661 (1991).

[8] D. J. Wineland et al., quant-ph/0212079 (2002).

[9] J. I. Cirac and P. Zoller, Phys. Rev. Lett. 74, 4091 (1995).

[10] A. Sörensen and K. Mølmer, Phys. Rev. Lett. 82, 1971 (1999).

[11] S. Osnaghi et al., Phys. Rev. Lett. 87, 037902 (2001).

[12] G. H. Golub and C. F. van Loan, Matrix Computations (Johns Hopkins University Press, 1996), 3rd ed.

[13] F. Verstraete and H. Verschelde, Phys. Rev. Lett. 90, 097901 (2003).

[14] W. Dür, J. I. Cirac, and R. Tarrach, Phys. Rev. Lett. 83, 3562 (1999).

[15] A. Peres, Phys. Rev. Lett. 77, 1413 (1996).

[16] M. Horodecki, P. Horodecki, and R. Horodecki, Phys. Rev. Lett. 78, 574 (1997).

[17] J. I. Cirac et al., Phys. Rev. Lett. 86, 544 (2001). 
[18] Fortschritte der Physik 48, issues 9 to 11 (2000).

[19] Experiments often produce entanglement via indirect interactions. In ion-traps, electronic states of the ions interact via phonon modes; in cavities, atoms are entangled via interactions with photons in the cavity. (See e.g. [18].)

[20] We say a state $\rho_{a b c}$ is separable with respect to the partition $(a b)-c$ iff $\rho_{a b c}=\sum_{k}\left|\varphi_{k}\right\rangle_{a b}\left\langle\varphi_{k}|\otimes| c_{k}\right\rangle\left\langle c_{k}\right|$, and is entangled otherwise. 